DOI: 10.17234/SRAZ.65.50

UDK: 811.131.1'282.2(497.5 Rijeka)(091)

\title{
Indagini preliminari sulla storia della varietà italoromanza di Fiume*
}

\author{
Maja Đurđulov \\ Iva Peršić \\ Università degli Studi di Fiume / Sveučilište u Rijeci \\ Facoltà di Lettere e Filosofia / Filozofski fakultet u Rijeci \\ mdjurdjulov@uniri.hr, \\ iva.persic@uniri.hr
}

\begin{abstract}
Nel presente contributo verranno presentati i primi risultati del progetto Indagini per una storia della varietà italoromanza usata a Fiume, sovvenzionato dall'Università di Fiume, il quale ha come obiettivo principale lo studio della varietà italoromanza utilizzata a Fiume attraverso l'uso delle fonti documentarie. Il progetto si propone di studiare le fonti disponibili per tracciare e descrivere dal punto di vista storico, culturale e linguistico il panorama italoromanzo a Fiume, e di capire che cosa caratterizza dal punto di vista linguistico la varietà italoromanza usata in questo territorio, dal momento in cui è attestata fino ai giorni nostri, con lo scopo di tracciare una 'storia dell'italiano di Fiume'. Il presente contributo riguarda la prima parte del progetto, incentrata sulla ricognizione e la catalogazione, lo studio e il commento di alcuni documenti scritti in italiano e nelle sue varietà, prodotti a Fiume in vari periodi storici.
\end{abstract}

Parole chiave: italiano, fiumano, volgare, Fiume, storia

\section{Introduzione}

La città di Fiume sorge sul mare, in una posizione favorevole sia dal punto di vista geografico sia da quello economico, nell'intersezione di diverse vie naturali che conducono verso Trieste (e quindi verso l'Italia, passando per la Slovenia), verso la costa orientale della penisola istriana, verso il litorale dalmata e verso l'entroterra croato e, poi, ungherese. La città è, inoltre, attraversata da un fiume che in passato è servito da confine amministrativo-politico, ma non ha mai bloccato gli scambi etnici, linguistici e culturali tra le popolazioni che lo circondavano. Grazie alla sua posizione geografica, Fiume si è profilata come una delle città portuali più importanti sulla costa orientale dell' Adriatico, il che ha ulteriormente

* Il presente contributo è stato sovvenzionato dall'Università degli Studi di Fiume (unirihuman-18-169). 
potenziato i suddetti scambi, i quali, a loro volta, hanno portato alla creazione di «un crogiuolo di stirpi e di lingue» (Ramous 1975: 120).

La lunga e complessa storia di Fiume ${ }^{1}$ verrà menzionata solo marginalmente per inquadrare il periodo al quale risalgono i documenti esaminati e per illustrare le ragioni della ricchezza culturale e linguistica fiumana. I primi insediamenti sul territorio risalgono al Paleolitico, tra le prime popolazioni che lo abitavano figurano i Liburni, ai quali si aggiunsero gli Avari e gli Slavi tra la fine del VI e gli inizi del VII secolo. Il territorio fu in seguito, agli inizi dell'XI secolo, dominato dai Franchi, ma mantenne una certa autonomia. Questa caratteristica, di volere conservare la propria autonomia, sarà tipica della città di Fiume anche nei secoli a venire.

Secondo Stelli (2017: 16), «nei tre secoli che vanno dal IX all'XI si delineano con chiarezza i fattori fondamentali che determineranno in vario modo la storia di Fiume fino ai giorni nostri: il nucleo persistente della popolazione originaria latina e latinizzata, il mondo croato, il mondo germanico, il Regno d'Ungheria e, infine, Venezia». Anche se il dominio della Serenissima su Fiume fu per lo più di tipo indiretto, la sua influenza culturale e linguistica fu costante. Con l'entrata della città nel sistema feudale carolingio, dal IX secolo, divenne sempre più forte pure l'influenza germanica.

Tutti questi cambiamenti e la mescolanza di etnie, culture e lingue diverse hanno lasciato tracce, più o meno evidenti, anche sulla varietà italoromanza parlata a Fiume. Uno degli obiettivi del progetto di cui questo contributo fa parte è proprio quello di cercare di fare chiarezza su quanto vi abbiano inciso gli scambi commerciali e culturali con la sponda occidentale dell' Adriatico.

Stelli (2017: 31) afferma che i mercanti fiumani appresero da quelli veneziani e marchigiani le modalità di gestione dei registri e libri contabili, lo stile di scrittura dei documenti legali e altri tipi di testi legati al mondo degli affari. I primi documenti scritti finora conosciuti attestanti l'uso di una varietà italoromanza a Fiume sono di tipo amministrativo e risalgono al XV secolo. All'epoca la lingua ufficiale era il latino ma nei documenti che verranno trattati di seguito si trovano tracce della variante italoromanza locale, fatto che presuppone la presenza di un nucleo di parlanti che utilizzavano un qualche tipo di italiano volgare.

\section{Documenti reperiti}

La prima fase del progetto Indagini per una storia della varietà italoromanza usata a Fiume, sovvenzionato dall'Università di Fiume, comprende l'individuazione e la catalogazione dei documenti scritti interamente o in parte in italiano o nella sua varietà fiumana, nonché lo studio degli stessi. Oltre ai documenti presenti nell'Archivio di Stato di Fiume, molte fonti documentarie si trovano nella

1 Per maggiori informazioni sulla complessa e variamente interpretata storia di Fiume si rimanda ad esempio a: AA.VV. 1988; Fried 2005; Kobler 1896; Simonić 2015; Stelli 2017; Žic 2007.

2 Per maggiori informazioni sulla stessa si rimanda al sito dell'associazione http://www. fiume-rijeka.it/. 
Biblioteca della Società di Studi Fiumani a Roma. ${ }^{2}$ In quest'ultima sono stati rinvenuti i documenti che verranno elencati di seguito, raggruppati per secolo in cui sono stati prodotti, e sui quali sono state eseguite le prime analisi.

I primi documenti reperiti nei quali compare una varietà italoromanza sono stati redatti verso la metà del XV secolo e sono presenti nel cosiddetto libro del cancelliere (Liber Civilium sive Notificationum). ${ }^{3}$ Questi comprendono vari rogiti, atti pubblici e scritture private, nonché le ordinanze proclamate ai cittadini dai banditori pubblici. Il cancelliere Antonio di Francesco de Reno è l'autore del libro contenente quelle che solitamente vengono citate come prime testimonianze del fiumano. ${ }^{4}$ Si tratta di due trascrizioni risalenti al 16 aprile 1440 e al mese di ottobre del 1443, rispettivamente di un compromesso e di una sentenza. Segue poi il documento più studiato tra questi, il cosiddetto Calmiere del pesce del $1449 .{ }^{5} \mathrm{Di}$ quattro anni precedente è il manoscritto Lettera del capitano e dei giudici di Fiume alla Città di Cividale, del quale a Roma viene conservata una copia, mentre l'originale si trova a Udine nella Raccolta Conte Orso. Infine, risale al 29 dicembre 1457 la trascrizione dell'inventario del Duomo di Fiume. ${ }^{6}$

Nella biblioteca romana consultata è stato rinvenuto un unico documento scritto nel XVI secolo in italiano. Si tratta di una raccolta di trascrizioni (datata 1527-1530) in italiano degli Statuti concessi al comune di Fiume da Ferdinando I nel MDXXX, originariamente scritti in latino e vigenti fino agli inizi del XIX secolo.?

Se mancano documenti del XVII secolo, quelli del XVIII presenti nella Biblioteca della Società di Studi Fiumani sono numerosi. Il documento più antico risale al 1754 e si tratta di una lettera relativa alla causa mossa dal barone Domenico de Corrado contro Valentino Defranceschi. Seguono due testi a stampa in versione bilingue (tedesco e italiano), del 1757 e del 1760, relativi al dazio del vino, al diritto di cittadinanza e all'educilio di vino alla minuta. Altri tre documenti manoscritti di atti notarili riguardano delle vendite, transazioni e permute tra parti diverse. Infine, del 1785 è il manoscritto intitolato Nuovo regolamento per li sensali, della Città, e Porto Franco di Fiume, e di tutto il Littorale Ungarico.

Il XIX secolo è decisamente il periodo più ricco per quanto concerne i documenti rinvenuti. Si tratta di 17 testi a stampa, di cui la maggior parte contiene delle norme relative alla vita pubblica di Fiume con le quali si regolavano la fabbricazione di edifici, i dazi sui vini e sugli altri liquidi, la bollatura dell'oro e dell'argento, l'operato dei mercati pubblici e del mercato del pesce, le operazioni di scarico e carico dei bastimenti, le misure da adottare relative al colera, e l'illuminazione a gas in città. Inoltre, alcuni documenti fanno capo a questioni politiche, come la richiesta per la

3 L'argomento è stato trattato da Anna Rinaldin (Uno studio linguistico dei primi documenti volgari di Fiume (prima metà XV sec.)) e presentato al Congresso dell' Atlante Linguistico Mediterraneo (ALM) tenutosi a Grado dal 30 settembre al 2 ottobre 2019. Gli atti del convegno sono in corso di pubblicazione.

4 Cfr. Gigante (1932).

5 Cfr. Deanović (1965: 105-108).

6 Cfr. Fest (1913: 67-68).

7 Cfr. Silvino Gigante (1910). 
reincorporazione di Fiume all'Ungheria da parte delle autorità cittadine del 1861, un memoriale per l'Imperatore da parte del Consiglio Comunale del 1863, lo Statuto della libera città di Fiume e del suo distretto del 1872, con la traduzione ungherese e il discorso pronunciato dal Conte Governatore in occasione della ricorrenza della pubblicazione del documento. Infine, alcuni documenti degli anni ‘20 e`30 del XIX secolo riguardano l'operato di due istituzioni cittadine, l'Instituto Generale de' poveri e la Casa di Lavoro, ovvero i loro regolamenti e bilanci.

\section{Alcuni esempi}

Pur trattandosi ancora di un'indagine preliminare, verranno presentati di seguito alcuni esempi tratti dai documenti finora raccolti.

Il primo esempio da menzionare è il secondo più antico documento di cui siamo a conoscenza, presente nel quaderno notarile del notaio e cancelliere fiumano Antonio di Francesco de Reno di Modena, il quale teneva un libro nel quale copiava gli atti pubblici e le scritture private da lui rogate. Il libro raccoglie documenti risalenti al periodo dal 1436 al 1461 ed è quasi interamente scritto in latino, tranne quattro documenti che sono i testi in italiano più antichi di cui abbiamo traccia nell'area di Fiume.

La parte esaminata, in particolare, è una sentenza, scritta in latino, all'interno della quale si trova la copia del compromesso tra i giudici Andrea de Crastouica e Quirino Spincich di Castua del 26 ottobre 1443, scritto in volgare. Il testo, scritto da Adamo de Antonio da Fermo, riporta i nomi degli arbitri per ciascuna delle due parti, le quali, a loro volta, si impegnano a non contraddirli.

L'inizio del documento riporta:

Al nome de dio A die 26 de otobre del 1443. Sia noto e manifesto a çascaduno che vedera ouer leçara questo presente scrito como çudexe Andrea de Crastouica e çudexe quirino spinciçich de ogne loro diferencia e dogne loro fato e raxone auessero abuto a fare in seme per fina aldie presente fano compromeso e comprometeno da cordo e de loro spontanea voluntade et eleçeno per arbitri arbitradori e compositori li infrascripti Çoe çudexe Andrea predicto eleçe per sua parte li nobili homini Antonio de Terençi da pesaro e çudexe Nicholò de Antonio da Fiume El dito çudexe quirino spinçich Eleçe zudexe Moro da Fiume e zudexe Antonio de Castoa che possano de raxone e de fato sentenciare achunciare e arbitrare tra de loro come meglio a loro parera [...] (Gigante, 1910: 127).

Emergono fin da subito alcune forme verbali che presentano caratteristiche morfologiche di area settentrionale, in particolare veneta: parera, guadagnara, avemo sentenciado, sentenciemo (nel documento appare pure la forma sentenciamo), dibia dare.

Inoltre, sono presenti numerosi casi di scempiamenti i quali, oltre che un naturale segnale della problematica codificazione delle doppie nel XV secolo, potrebbero essere un ulteriore indizio dell'influenza del parlato regionale dell'area veneta: otobre, scrito, diferencia, fato, fano, facio, compromeso, comprometono, 
prometendo, farano, contradire, soto, contradirà, ala, diferencia, quatrocento, setanta, tuta, tute, metergli, asignati, siche, caualo, vintioto, soma, fradelo.

Oltre agli esempi appena citati, è possibile trovare occasionalmente alcuni casi isolati di altre forme di area settentrionale, come l'avverbio suxo (= su) e il sostantivo fradelo il quale, presente anche nel primo documento in lingua italiana attestato a Fiume, ovvero il compromesso di Veglia, è una forma ampiamente attestata nell'area veneta, trentina, emiliana ma anche lombarda. Infine, sono da evidenziare le diverse forme della parola giudice (çudexe e zudexe), anch'esse forme dell'Italia settentrionale.

Il secondo testo è invece del XVIII secolo, più precisamente del 1785, ed è intitolato Nuovo regolamento per li sensali, Della Città, e Porto Franco di Fiume, e di tutto il Littorale Ungarico. Il testo regolava le attività dei sensali, cioè dei mediatori tra venditori e acquirenti in affari commerciali, in particolare nelle contrattazioni di prodotti agricoli e di bestiame, e ne stabiliva le tariffe. Anche in questo documento sono da segnalare le scelte ortografiche: innanzitutto gli scempiamenti, comunque meno evidenti rispetto al documento precedente, come sudetta, legitimi, ecedente, illegitime, metali, maritime, annulato, ma anche ipercorrettismi, ovvero parole in cui vengono inserite indebitamente delle consonanti doppie, come littorale (ma anche litorale), diffettosi, cottoni fillati, setta (ma anche sete, al plurale), nolleggi.

Dal punto di vista lessicale, risulta interessante segnalare il termine Pottasch, che compare nella parte relativa alla tariffa dei sensali, in cui troviamo un elenco di beni con accanto la percentuale che rimaneva ai sensali per il loro servizio. Il termine Pottasch è probabilmente una variante del tedesco Pottasche ed era il nome popolare del carbonato di potassio. Il termine italiano, potassa, è attestato solo dal 1791 e deriva dal francese potasse che, a sua volta, deriva dall'olandese potasch (Cortelazzo/Zolli 1999, s.v. potàssa).

\section{Conclusione}

Quale forma linguistica presentano i testi esaminati? Nei documenti più antichi emerge una maggiore coloritura dell'italiano settentrionale, con alcuni elementi veneziani, ma anche bolognesi, padovani ecc. Ciò conferma i contatti linguistici e culturali, oltre che commerciali, di Fiume con la penisola italiana. I testi più recenti, invece, presentano una maggiore uniformità linguistica e ortografica ma nemmeno essi sono privi di «spie» che rivelano la loro origine geografica. I testi presentati sono solo una piccola parte del corpus di documenti fiumani che sarà composto anche dai documenti presenti in altre sedi, come ad esempio l'Archivio di Stato di Fiume. Con ulteriori indagini si auspica di fare maggiore chiarezza su questa varietà italoromanza, in particolare sulla sua evoluzione nel tempo, e di integrare in questo modo gli studi (socio)linguistici esistenti anche in merito allo stato di salute attuale dell'idioma e alla sua salvaguardia. 


\section{Bibliografia}

AA.VV. (1988). Povijest Rijeke, Rijeka: Skupština općine Rijeka, Izdavački centar Rijeka.

Cortelazzo, Manlio / Zolli, Paolo (1999). Dizionario etimologico della lingua italiana, II ed., [a cura di Manlio Cortelazzo e Michele A. Cortelazzo], Bologna: Zanichelli.

Deanović, Mirko (1965). Un calmiere per il pesce del 1449 a Fiume (Rijeka), in: Bollettino dell'Atlante Linguistico Mediterraneo, 7, pp. 105-108.

Fest, Alfredo (1913). Fiume nel secolo XV, in: Bullettino della Deputazione fiumana di storia patria, III, pp. 5-138.

Fried, Ilona (2005). Fiume. Città della memoria. 1868-1945 [trad. Lavinia Sandor e Ilona Fried], Udine: Del Bianco Editore.

Gigante, Silvino [a cura di] (1910). Monumenti di storia fiumana - Statuti concessi al comune di Fiume da Ferdinando I nel MDXXX, Fiume: Stabilimento litotipografico di E. Mohovich, 1910.

Gigante, Silvino [a cura di] (1932). Monumenti di storia fiumana - Libri del cancelliere, Fiume: Stabilimento tipografico de "La Vedetta d'Italia" S.A., 1912-1932.

Kobler, Giovanni (1896). Memorie per la storia della liburnica città di Fiume scritte dal Fiumano Giovanni Kobler, Fiume: Stabilimento Tipo-litografico Fiumano di Emidio Mohovich.

Ramous, Osvaldo (1975). Rapporti culturali tra le Venezie e la costa orientale dell'Adriatico, in: Unità e diffusione della civiltà veneta: relazioni e comunicazioni del Convegno degli scrittori veneti. Gorizia, ottobre 1974 [a cura di Ugo Fasolo e Nereo Vianello], Venezia: Associazione degli scrittori veneti.

Simonić, Ante (2015). Rijeka vremena. Drevno susretište zavičajnog i svjetskog. Knjiga proa, Zagreb: HAZU et alii.

Simonić, Ante (2015). Rijeka velikog uzleta. Zavodljivost starog i izazov novog. Knjiga druga, Zagreb: HAZU et alii.

Simonić, Ante (2015). Rijeka novih nadanja. Stvaranje poželjne sadašnjosti i okrenutost budućnosti. Knjiga treća, Zagreb: HAZU et alii.

Stelli, Giovanni (2017). Storia di Fiume. Dalle origini ai giorni nostri, Pordenone: Edizioni Biblioteca dell'Immagine.

Žic, Igor (2007). Breve storia della città di Fiume, Fiume: Adamić.

\section{Sitografia}

Società di Studi Fiumani, <http://www.fiume-rijeka.it/>, ultimo accesso: 25 giugno 2020. 


\section{Uvodna istraživanja o povijesti italoromanskoga jezičnog varijeteta u Rijeci}

U ovom će se radu predstaviti prvi rezultati projekta Istraživanjem do povijesnog pregleda italoromanskog jezičnog varijeteta u Rijeci, koji financira Sveučilište u Rijeci, a čiji je primarni cilj proučiti kroz dokumentarne izvore italoromanski jezični varijetet u uporabi u Rijeci. Projektom se žele istražiti dostupni dokumenti kako bi se odredila i opisala s povijesnog, kulturnog i jezičnog gledišta italoromanska jezična panorama Rijeke, te razjasnila jezična obilježja tog lokalnog govora, počevši od njegovih prvih dokumentiranih pisanih tragova pa sve do danas, s ciljem utvrđivanja povijesti talijanskoga jezika u Rijeci.

Ovaj rad predstavlja prvi dio samoga projekta, koji uključuje popisivanje i katalogizaciju te analizu i opis dokumenata napisanih na talijanskom jeziku ili nekom njegovom varijetetu, nastalih u Rijeci u različitim povijesnim razdobljima.

Ključne riječi: talijanski jezik, fijumanski dijalekt, narodni talijanski jezik, Rijeka, povijest 
